\title{
Photonic band gaps and defects in two dimensions: Studies of the transmission coefficient
}

\author{
M. Sigalas, C. M. Soukoulis, E. N. Economou, ${ }^{*}$ C. T. Chan, and K. M. Ho \\ Ames Laboratory and Department of Physics and Astronomy, Iowa State University, Ames, Iowa 50011
}

(Received 21 May 1993)

\begin{abstract}
Using the transfer-matrix technique for the propagation of electromagnetic waves in dielectric structures, we calculate the transmission coefficient versus the frequency of the incident wave for different polarizations in two-dimensional periodic and/or random arrangements of dielectric cylinders. This technique has been applied to cases where the plane-wave method fails or becomes too time consuming, such as when the dielectric constant is frequency dependent or has a nonzero imaginary part, and when defects are present in an otherwise periodic system. For all the cases studied, the results compared well with experiment.
\end{abstract}

\section{INTRODUCTION}

There is a growing interest in the studies of the propagation of electromagnetic (EM) waves in periodic (both in two and three dimensions) and/or random dielectric structures (photonic band structures). The existence, in periodic media, of a frequency gap where the propagation of EM waves is forbidden for all wave vectors, can have a profound impact on several scientific and technical disciplines. ${ }^{1,2}$ It is therefore very important to obtain three-dimensional (3D) and $2 \mathrm{D}$ periodic structures that possess a full photonic band gap. Theoretical calculations of Ho, Chan, and Soukoulis in 3D have shown that periodic dielectric materials with a diamond ${ }^{3}$ or diamondlike structure ${ }^{4}$ can indeed have photonic band gaps. One of these structures, the "three-cylinder structure" which consists of three sets of cylinders drilled into a dielectric material at 35.26 degrees off normal, has been fabricated $^{5}$ in the millimeter length scale and shown to possess full photonic gap in the microwave region, in agreement with the predictions ${ }^{4}$ of the theoretical calculations. Very narrow photonic band gaps have been found also $^{6}$ in a simple cubic geometry. For two-dimensional systems, ${ }^{7-9}$ theoretical studies ${ }^{7,8}$ have shown that a triangular lattice of air columns in a dielectric background is the best overall 2D structure, which gives the largest photonic gap with the smallest index contrast. In addition, it was demonstrated ${ }^{10-13}$ that lattice imperfections in $2 \mathrm{D}$ and/or 3D periodic arrays of a dielectric material can give rise to fully localized EM wave functions. Experimental investigations of the photonic band gaps have been mostly done ${ }^{10,12,13}$ at microwave frequencies because of the difficulty in fabricating ordered dielectric structures of optical length scales. The experimental results ${ }^{10,12,13}$ are in excellent agreement with theoretical calculations.

Most of the theoretical calculations ${ }^{1,3^{-9}}$ take into account the similarity that exists between electronic states in a periodic potential and the EM waves in a periodic dielectric medium. Techniques, such as the plane wave developed for electronic-structure calculations, have been applied to calculate the band structure for EM waves propagating in either $2 \mathrm{D}$ or $3 \mathrm{D}$ periodic dielectric structures. The plane-wave expansion technique for EM waves is now well developed. Most of the techniques concentrate on the calculation of the dispersion of the photon bands in the infinite periodic structure, while experimental investigations focus mainly on the transmission of electromagnetic waves through a finite slab of the photonic band-gap material patterned in the required periodic structure. Even with the knowledge of the photon band structure, it is still a nontrivial task to obtain the transmission coefficient for comparison with experiment. Another quantity important for the design of photonic band-gap experiments and devices is the attenuation length for incident electromagnetic waves inside the photonic band gap. Another topic of interest is the behavior of impurity modes associated with the introduction of defects into the photonic band-gap structure. While this problem can be tackled within a plane-wave approach using the supercell method ${ }^{10,11}$ in which a single defect is placed within each supercell of an artificially periodic system, the calculations require a lot of computer time and memory. Recently, Pendry and MacKinnon ${ }^{14}$ introduced a complementary technique of studying photonic bandgap structures. Their method has the advantage that the transmission coefficients and attenuation coefficients for incident electromagnetic waves of various frequencies can be obtained directly from the calculations. Their method can also be efficiently used in cases when the plane-wave expansion method fails or becomes too time consuming. In particular, when the dielectric function $\epsilon$ is frequency dependent, or when $\epsilon$ has large imaginary values, Fourier expansion methods are not useful. Disordered systems and periodic systems with imperfections can be easily studied by the method. The band structure techniques usually calculate the frequency $\omega$ given a real wave vector $\mathbf{k}$ and a comparison with a transmission experiment where an EM wave of fixed frequency $\omega$ incident on a dielectric sample is not trivial. The incident EM wave excites all bands at that particular frequency. This method ${ }^{14}$ for a given $\omega$ calculates all the bands $\mathbf{k}(\omega)$. 
In this paper we report calculations for the photonic band structure of periodic $2 \mathrm{D}$ arrays of cylindrical dielectric scatterers, as well as cases where single or multiple defects are introduced. Comparison of the calculated transmission coefficient with experimental studies is excellent. The role of the absorption [i.e., a nonzero imaginary value of $\epsilon(\mathbf{r})$ ] of the dielectric scatterers is also studied. It is clearly shown that the photonic band gaps get smaller as the imaginary part of the dielectric function gets larger. The transmission coefficient calculations of the disordered case compare successfully with the experiments and are capable of several predictions for arbitrary dielectric structures, with real and imaginary values of the dielectric constant. This method was also tested and used for $3 \mathrm{D}$ dielectric structures ${ }^{15}$ with satisfactory agreement with experimental studies.

In Sec. II we describe the methods of calculating the transmission coefficient. In Sec. III we present and discuss the results and in Sec. IV we summarize the conclusions of this work.

\section{METHODS OF CALCULATIONS}

We are interested in the propagation of EM waves in a system that consists of a periodic and/or a random array of infinitely long parallel, identical dielectric rods, characterized by a dielectric constant, $\epsilon_{a}$, embedded in a background dielectric material characterized by a dielectric constant, $\epsilon_{b}$. The rods are assumed to be parallel to the $x_{3}$ axis. The intersections of the rods with the $x_{1} x_{2}$ plane form a periodic $2 \mathrm{D}$ structure. We are mostly interested in studying the case where the EM waves propagate in a plane perpendicular to the axes of the dielectric rods, i.e., in the $x_{1} x_{2}$ plane. Two polarizations of the EM field are considered: first, the $E$ polarization, in which the electric field vector is parallel to the $x_{3}$ axis, and second, the $H$ polarization, in which the magnetic field vector is parallel to the $x_{3}$ axis. The starting point is Maxwell's equations

$$
\boldsymbol{\nabla} \times \mathbf{E}=i(\omega / c) \mathbf{E}, \quad \boldsymbol{\nabla} \times \mathbf{H}=-i(\omega / c) \epsilon(\mathbf{r}) \mathbf{E},
$$

where the dielectric constant $\epsilon(\mathbf{r})$ is position dependent, and we seek solutions of Maxwell's equations which have the form $\mathbf{E}(\mathbf{r}) \exp (-i \omega t)$.

For the case of $E$ polarization $\mathbf{E}(\mathbf{r})=(0,0, E)$ and $\mathbf{H}(\mathbf{r})$ $=\left(H_{1}, H_{2}, 0\right)$, Maxwell's equations give that

$$
\begin{gathered}
\frac{\partial E}{\partial x_{1}}=-\frac{i \omega}{c} H_{2} ; \quad \frac{\partial E}{\partial x_{2}}=\frac{i \omega}{c} H_{1} \\
\frac{\partial H_{2}}{\partial x_{1}}-\frac{\partial H_{1}}{\partial x_{2}}=-\frac{i \omega}{c} \epsilon E .
\end{gathered}
$$

The equation for the electric field $E$ obtained by eliminating $H_{1}$ and $H_{2}$ from Eqs. (2a) and (2b) can be written as

$$
\left(\frac{\partial^{2}}{\partial x_{1}^{2}}+\frac{\partial^{2}}{\partial x_{2}^{2}}\right) E+\epsilon \frac{\omega^{2}}{c^{2}} E=0
$$

and by discretizing Eq. (3), one obtains

$$
\begin{aligned}
\left(4-\frac{\omega^{2} b^{2}}{c^{2}} \epsilon_{i, j}\right) E_{i, j}-E_{i, j-1} & -E_{i-1, j} \\
& -E_{i, j+1}-E_{i+1, j}=0
\end{aligned}
$$

where the indices $i$ and $j$ denote the $x_{1}$ and $x_{2}$ axis, respectively, and $b$ is the distance between neighboring nodes in a uniform discrete $2 \mathrm{D}$ mesh. Equation (3) can be solved by the plane-wave expansion method when the dielectric function $\epsilon$ is a periodic function. This is the technique that most of the theoretical work in $2 \mathrm{D}$ has used..$^{1,2,7-9}$ However, Eq. (4) is exactly equivalent to the well-studied problem of the tight-binding model of electronic localization. ${ }^{16-18}$ In particular, the most successful method in obtaining the localized or extended nature of the wave functions in disordered and/or periodic electronic systems is the transfer-matrix technique. Equation (4) can be solved by the transfer-matrix technique, ${ }^{18}$ where the electric fields $E$ on one side of a structure are related to those on the other. This way the transmission coefficient through a particular dielectric arrangement can be calculated. In addition, if the particular dielectric arrangement is periodic, the eigenvalues of the transfer matrix give the band structure of the system. Notice that, in the propagation of EM waves in $2 \mathrm{D}$ dielectric structures, the electric and magnetic fields are decoupled and one has a scalar problem [see Eq. (4) for the $E$ polarization] as in the case of the tight-binding model for the propagation of electrons. For the 3D case, the electric and magnetic fields are not decoupled ${ }^{14}$ and one has a more complex transfer matrix to iterate. ${ }^{15}$

For the case of $H$ polarization $\mathbf{H}(\mathbf{r})=(0,0, H)$ and $\mathbf{E}(\mathbf{r})=\left(E_{1}, E_{2}, 0\right)$, Maxwell's equations take the form

$$
\begin{gathered}
\frac{\partial H}{\partial x_{1}}=\frac{i \omega}{c} \epsilon E_{2} ; \quad \frac{\partial H}{\partial x_{2}}=-\frac{i \omega}{c} \epsilon E_{1}, \\
\frac{\partial E_{2}}{\partial x_{1}}-\frac{\partial E_{1}}{\partial x_{2}}=\frac{i \omega}{c} H .
\end{gathered}
$$

The equation for the magnetic field $H$ obtained by eliminating $E_{1}$ and $E_{2}$ from Eqs. (5a) and (5b) can be written as

$$
\frac{\partial}{\partial x_{1}}\left(\frac{1}{\epsilon} \frac{\partial H}{\partial x_{1}}\right)+\frac{\partial}{\partial x_{2}}\left(\frac{1}{\epsilon} \frac{\partial H}{\partial x_{2}}\right)+\frac{\omega^{2}}{c^{2}} H=0
$$

and by discretizing Eq. (6) one obtains

$$
\begin{array}{r}
\left(\epsilon_{i+1, j}^{-1}+2 \epsilon_{i, j}^{-1}+\epsilon_{i, j+1}^{-1}-\frac{\omega^{2} b^{2}}{c^{2}}\right) H_{i, j}-\epsilon_{i, j+1}^{-1} H_{i, j+1} \\
-\epsilon_{i, j}^{-1} H_{i, j-1}-\epsilon_{i+1, j}^{-1} H_{i+1, j}-\epsilon_{i, j}^{-1} H_{i-1, j}=0
\end{array}
$$

where the indices $i$ and $j$ denote the $x_{1}$ and $x_{2}$ axis, respectively. Again Eq. (6) as Eq. (3) for the $E$ polarization can and has been solved ${ }^{1,2,7-9}$ by the plane-wave expansion method. Equation (7) is also equivalent to a tight-binding model of electronic localization; however, 
Eq. (7) is of the off-diagonal type ${ }^{17}$ of disorder, while Eq. (4) is of diagonal type of disorder. ${ }^{16-18}$ In the electronic localization it is well known ${ }^{17}$ that the off-diagonal case gives extremely large localization lengths and, therefore, gives transmission coefficients very close to unity even for localized states. Indeed, we find that for the $H$ polarization (off-diagonal case for the corresponding electronic problem), one needs to be extremely careful to obtain the correct photonic band gap. In addition, Eqs. (4) and (7) can be used to calculate the transmission coefficients for disordered dielectric structures, as well as for periodic dielectric structures with one or more imperfections. The treatment of frequency dependent dielectric constant $\epsilon$, as well as of an imaginary part of $\epsilon$, can also be handled by the transfer-matrix technique.

\section{RESULTS AND DISCUSSION}

To demonstrate the efficiency and the capabilities of this method, we made a systematic comparison of our theoretical results with the experimental results of Refs. 2 and 12. The experimental system consists of arrays of dielectric cylindrical scatterers. The dielectric cylinders with radius $a=0.48 \mathrm{~cm}$, and dielectric constant $\epsilon=9$, form a square lattice with a lattice constant $d=1.27$ $\mathrm{cm}$. This corresponds to a filling ratio of $f=0.449$. The experiments were carried out at microwave frequencies ( 6-20 GHz). In Fig. 1(a) we present the theoretical results for the transmission coefficient versus frequency for the periodic square lattice with the propagation of EM waves being along the $(1,0)$ direction; there are eight layers of cylinders along this direction. Notice that the transmission coefficient is considerably reduced around three frequencies (at 5, 10, and $15 \mathrm{GHz}$ ), corresponding to the three photonic band gaps calculated by the planewave expansion method. In Fig. 1(a) we also present the results of the transmitted power versus frequency when the dielectric constant has a positive imaginary value describing the absorption of energy by the dielectric cylinders. This capability of the transfer-matrix technique is an important feature that is absent from the plane-wave method. Notice that as the imaginary part of $\epsilon$ increases, the transmitted power is reduced for all frequencies, as expected because of absorption. However, the reduction is larger when the frequency increases.

In Fig. 1(b) we present the results for the $H$ polarization. Notice that for the case with a real dielectric constant $\epsilon=9$, there is a photonic band gap around 11 $\mathrm{GHz}$ and its extent is $2 \mathrm{GHz}$. Thus, the second gap of the $E$ polarization coincides with the first gap of the $H$ polarization [see Fig. 1(b), solid line]; there are also gaps around 13 and $15 \mathrm{GHz}$. The effect of a nonzero value of the imaginary part of the $\epsilon$ on the transmitted power [see Fig. 1(b)] for the $H$ polarization is similar to that of the $E$ polarization. There is a strong reduction of the transmitted power at high frequencies. In Fig. 2 we present the results of the reflected power versus frequency for the $2 \mathrm{D}$ square lattice of dielectric cylinders for the two polar-
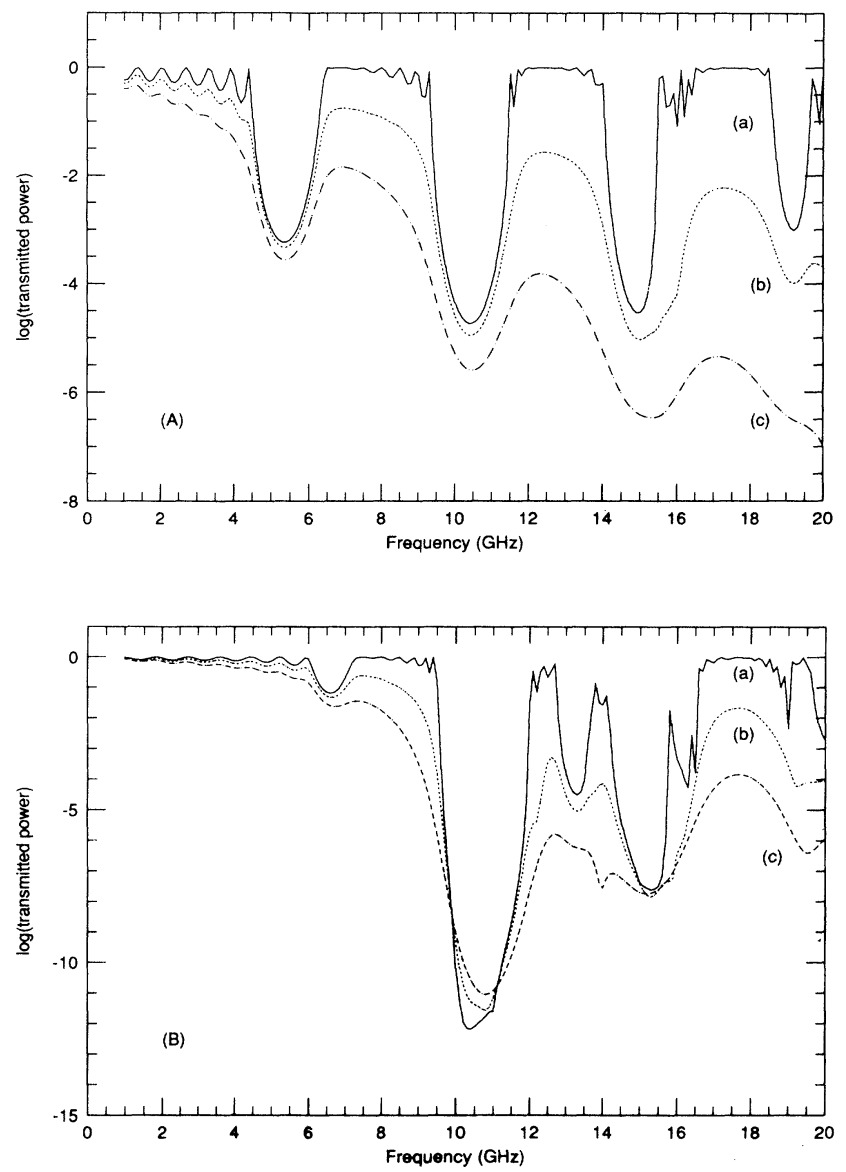

FIG. 1. The transmitted power vs frequency for a $2 \mathrm{D}$ square lattice consisting of cylinders with $f=0.449$ surrounded by air for $E$ - and $H$-polarized EM waves ( $A$ and $B$ panels, respectively) propagating along the $(1,0)$ direction (there are eight layers of cylinders along this direction); the real part of $\epsilon$ is 9 and the imaginary part is 0 (a); 0.4 (b); 1 (c); $d=1.27 \mathrm{~cm}$. Power is in arbitrary units.

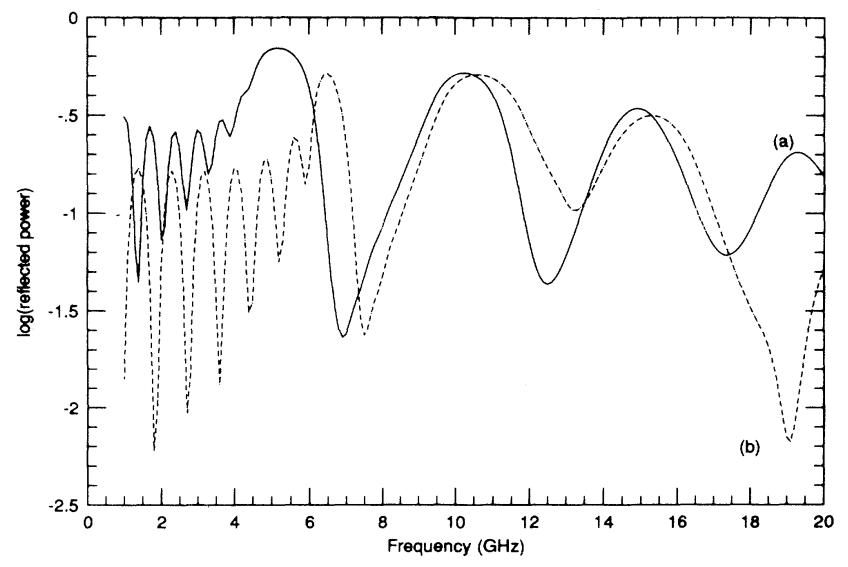

FIG. 2. The reflected power vs frequency in a similar system as in Fig. 1 for (a) $E$ - and (b) $H$-polarized EM waves; $d=1.27 \mathrm{~cm}, \epsilon=9+i$. Power is in arbitrary units. 
izations in the case where $\epsilon=9+1 i$, i.e., a relatively large value of the imaginary part of $\epsilon$. Notice there are many oscillations at low frequencies (see similar oscillations in Fig. 1) which are the result of the multiple scattering between the two surfaces of the material; for this reason they tend to disappear as the imaginary part of $\epsilon$ increases. It is also possible with our technique to calculate the reflection coefficient, which is the more relevant quantity when absorption processes become dominant. This transfer-matrix technique can be used to design structures with particular reflecting properties.

In polar crystals, the coupling between the transverse optical phonons and the transverse electromagnetic waves affects the propagation of EM waves in the material. This coupling can be described by the frequency dependent dielectric constant:

$$
\epsilon(\omega)=\epsilon_{\infty} \frac{\omega_{L}^{2}-\omega^{2}}{\omega_{T}^{2}-\omega^{2}}
$$

[in the present case we assume that the imaginary part of $\epsilon(\omega)$ is zero]. In this case, the dielectric material exhibits a gap between $\omega_{L}$ and $\omega_{T}$, where EM waves cannot propagate. We want to study how the two gaps interact in a periodic structure where the high dielectric material is a polar material characterized by the frequency dependent dielectric constant given in Eq. (8). In particular, we considered a 2D square lattice consisting of GaAs cylinders with filling ratio $f=0.449$ as in Fig. 1 ; for GaAs we have that $\epsilon_{\infty}=10.9, \omega_{L}=0.875 \times 10^{13} \mathrm{~s}^{-1}, \omega_{T}=0.812 \times$ $10^{13} \mathrm{~s}^{-1}$. GaAs is a material that can be used to create a 2D photonic band-gap structure in the visible region. In the homogeneous case $(f=1)$, the reflectance will be one for $\omega_{T}<\omega<\omega_{L}$ so there is a forbidden frequency band (FFB) between $\omega_{T}$ and $\omega_{L}$; this FFB appears also in the nonhomogeneous cases $(f \neq 1)$ as we can see in Fig. 3. The gaps will be affected only when they are close to the FFB region, for that reason we considered

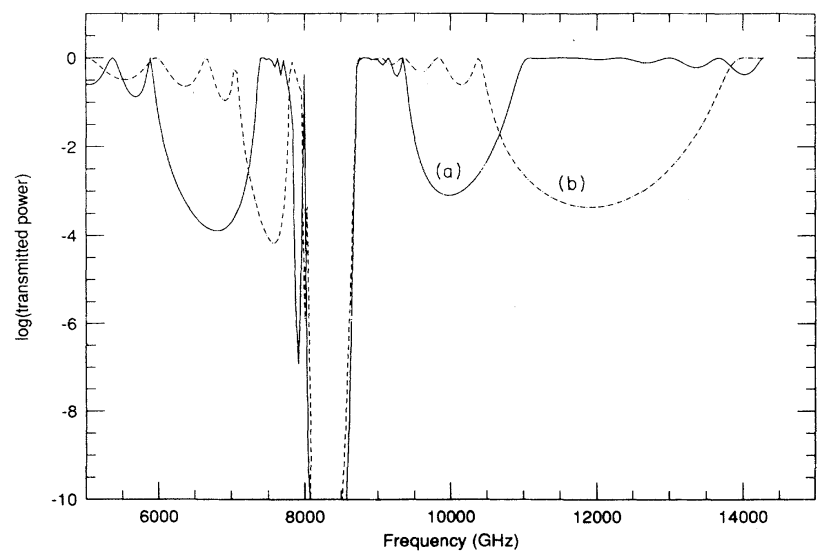

FIG. 3. The transmitted power vs frequency in a similar system as in Fig. 1 for $E$-polarized EM wave and two different lattice constants (a) $d=7.54 \mu \mathrm{m}$, (b) $5.65 \mu \mathrm{m}$. The dielectric constant is frequency dependent and is given by Eq. (8). lattice constants close to $6 \mu \mathrm{m}$ (the $f$ remains constant) so the frequency of the gap will be close to either $\omega_{L}$ or $\omega_{T}$. In Fig. 3 we present the results for two different lattice constants $(d=7.54 \mu \mathrm{m}$ and $d=5.65 \mu \mathrm{m})$ and $E$ parallel to the axis of the cylinders (the conclusions are the same for both $E$ and $H$ polarizations). For the 2D square lattice with $d=7.54 \mu \mathrm{m}$ and $f=0.449$ and $\epsilon=10.9$, the frequencies, where the first two gaps appear, are roughly at $0.8 \times 10^{4}$ and $1.6 \times 10^{4} \mathrm{GHz}$. This can be easily obtained from Fig. 1 since the frequency scales as $1 / d$. For $d=5.65 \mu \mathrm{m}$, the first two gaps are roughly at $1.1 \times 10^{4}$ and $2.2 \times 10^{4} \mathrm{GHz}$. However, as one can clearly see from Fig. 3, once the frequency dependent dielectric constant given by Eq. (8) is used, the positions of the gaps are strongly modified. Notice that for the $d=7.54$ $\mu \mathrm{m}$ case the first two gaps are now centered at around $0.65 \times 10^{4} \mathrm{GHz}$ and $0.95 \times 10^{4} \mathrm{GHz}$, while there is also a strong dip in the transmission coefficient just below $\omega_{T}$. Similar behavior can be seen for the $d=5.65 \mu \mathrm{m}$ case.

We now turn to the calculation of the transmitted power versus frequency for the cases where one or more cylinders have been removed from the otherwise periodic array of $2 \mathrm{D}$ dielectric cylinders. It is clearly seen experimentally ${ }^{1,2,12}$ that the removal of a single scatterer produces a highly localized EM defect mode. Experimental studies of Ref. 2 have followed the evolution of the defect states created by randomly removing an increasing number of cylinders from a square until $50 \%$ of the cylinders are removed. In Fig. 4, we present a sequence of transmission calculations of a periodic lattice with 162 sites $(9 \times 18)$ with an increasing number of cylinders removed. The dotted line in Fig. 4 shows the periodic case with no cylinders removed. This transmitted power versus frequency agrees with the results presented in Fig. 1(a) for the $E$ polarization. The results presented in Fig. 4 were calculated by iterating Eq. (4) with the introduction of a Green's function as was done for the electronic case. ${ }^{17}$ As the number of cylinders removed increases, one starts seeing an impurity state inside the photonic band gap, which broadens to form an impurity

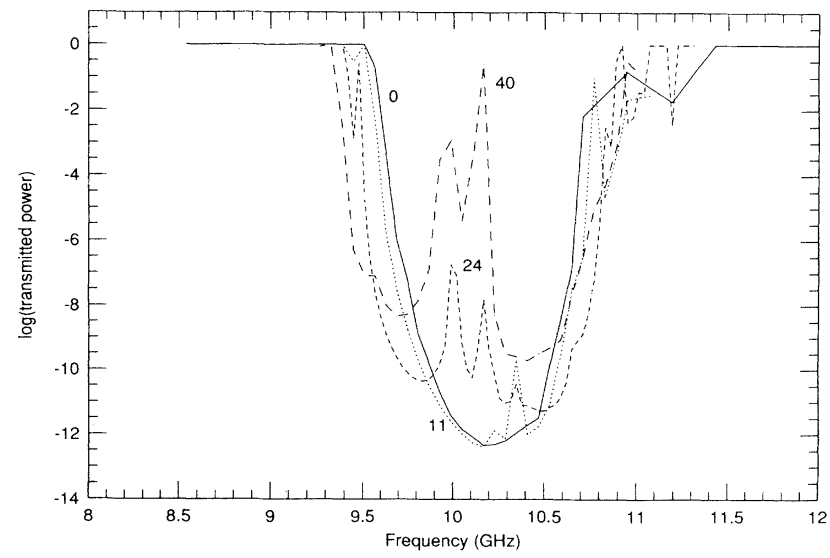

FIG. 4. The transmitted power vs frequency for a $2 \mathrm{D}$ square lattice consisting of $162(9 \times 18)$ cylinders with $f=0.449$ and $\epsilon=9$ surrounded by air; the dotted, solid, and dashed lines correspond to the perfect lattice, 11, 24, and 40 cylinders removed, respectively. 

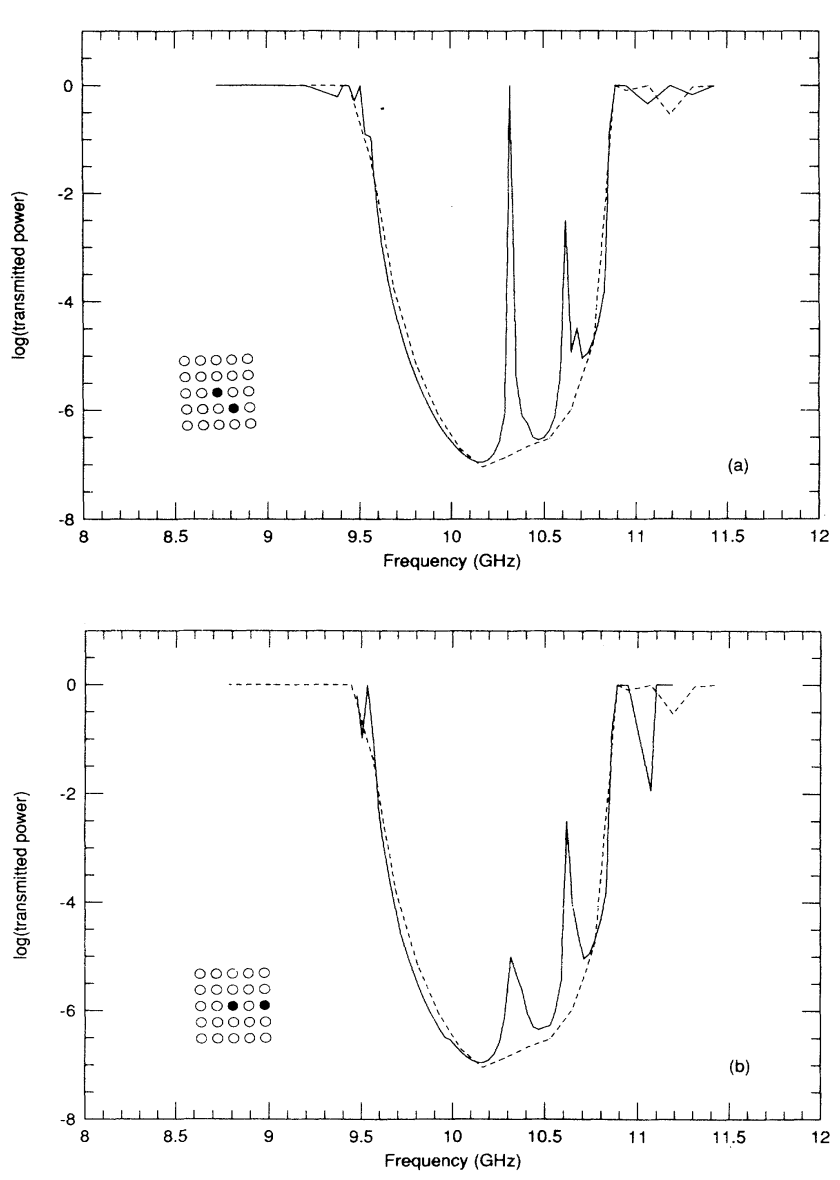

FIG. 5. The transmitted power vs frequency for a $2 \mathrm{D}$ square lattice consisting of $50(5 \times 10)$ cylinders with $f=0.449$ and $\epsilon=9$ surrounded by air; the solid and dotted lines correspond to the perfect lattice cylinders and the two neighboring (a) and non-neighboring defects (b) lattice, respectively.

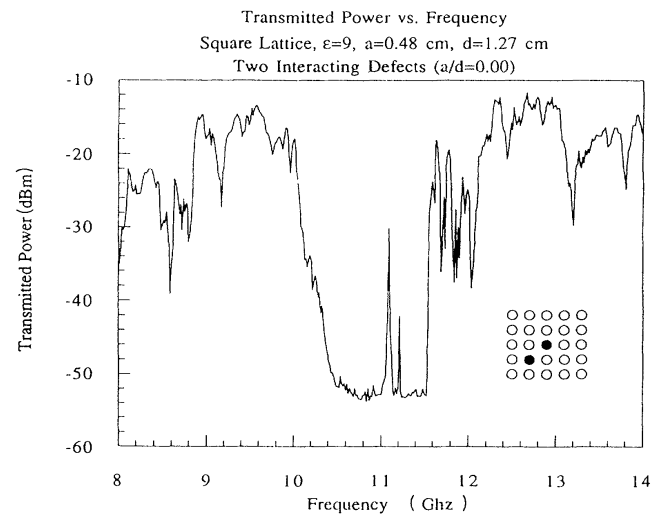

FIG. 6. The experimental power vs frequency for a $2 \mathrm{D}$ square lattice consisting of $(9 \times 18)$ cylinders with $f=0.449$ and $\epsilon=9$ surrounded by styrofoam $(\epsilon=1.04)$. Two cylinders are removed from near the center of the lattice. [Experiments by Schultz et al. (Ref. 12)]. band. This is clearly seen in Fig. 4, where a total of 11 then 24 cylinders and finally 40 out of the 162 cylinders are removed. Notice that in addition to the broadening of the impurity band in the photonic band gap (with two distinct peaks), there is an overall decrease in the transmitted power for all the frequencies studied. Another interesting result of our calculations is that the band edge moves outward as more defects (i.e., more cylinders are removed from the periodic structure) are introduced. This is similar to the behavior of the density of states in the electronic disordered systems. ${ }^{17}$ The slope of the transmission coefficient versus frequency might be used to obtain the Urbach tail for these disordered photonic band-gap structures. Our transmission coefficient studies agree reasonably well with the microwave experiments of Schultz et $a .^{12}$

It is interesting to see what happens when we remove two cylinders. We chose to work in a periodic lattice with $50(5 \times 10)$ cites because the number of removed cylinders is much smaller compared with the previous cases. We present the results in Fig. 5. Two sharp peaks [Fig. 5(a)] appear when we remove two second-nearestneighbor cylinders which are located close to the center of the lattice; the first peak is located close to the upper edge of the gap, while the second one is in the middle of the gap. These results are in excellent agreement with the experimental results of Refs. 2 and 12 (Fig. 6). Comparing Figs. 5(a) and 6 one sees that there is some small difference between theory and experiment about the exact positions of the peaks. We feel this is due to the fact that in theory we considered a square lattice with $(5 \times 10)$ cylinders, while the experiment was performed with $(9 \times 18)$ cylinders. In the case of third-neighbor defects [Fig. 5(b)], we also have two peaks which are located in nearly the same frequencies as in the previous case, but the second peak in the middle of the gap is much smaller. It seems that the impurity state which corresponds to that peak is more delocalized in the second-neighbor defect case as a result of the interaction of the two defect states. The overall agreement between the transmission coefficient studies and the microwave experiments supports the statement that these numerical studies of transmittance can be used reliably for predicting the behavior of yet untried structures.

\section{CONCLUSIONS}

In conclusion, we have presented a systematic study of the transmission coefficient versus frequency for $2 \mathrm{D}$ periodic and/or random arrangements of dielectric cylinders. The transfer-matrix technique, so often used in electronic tight-binding models, has been applied to the propagation of EM waves in dielectric structures. The method treats periodic dielectric arrangements, even when the dielectric constant is either frequency dependent or has a nonzero imaginary part. In addition, the transfer-matrix technique gives accurate results for the impurity modes created by either removing one or more cylinders from the otherwise periodic structure. The computer time needed for the calculation of the transmission coefficient for the states is much less than the supercell method ${ }^{11}$ used so 
far. Finally, this method can be easily extended to studies of $3 \mathrm{D}$ systems, with or without impurity modes.

\section{ACKNOWLEDGMENTS}

We thank S. Schultz for helpful discussions. Ames Laboratory is operated by the U.S. DOE by Iowa State
University under Contract No. W-7405-Eng 82. This work was supported by the Director for Energy Research, Office of Basic Energy Sciences, and Advanced Energy Projects, including a grant of computer time on the Cray computer at the Lawrence Livermore Laboratory, NSF Grant No. INT-9117356 and CEC Grant No. SCC* CT90-0020.
* Permanent address: Research Center of Crete-FORTH and Department of Physics, University of Crete, Heraklion, Crete, Greece.

${ }^{1}$ See the special issue of J. Opt. Soc. Am. B 10, 208-408 (1993).

${ }^{2}$ See the proceedings of the NATO ARW, Photonic Band Gaps and Localization, edited by C. M. Soukoulis (Plenum, New York, in press).

${ }^{3}$ K. M. Ho, C. T. Chan, and C. M. Soukoulis, Phys. Rev. Lett. 65, 3152 (1990).

${ }^{4}$ C. T. Chan, K. M. Ho, and C. M. Soukoulis, Europhys. Lett. 16, 563 (1991).

${ }^{5}$ E. Yablonovitch, T. J. Gmitter, and K. M. Leung, Phys. Rev. Lett. 67, 2295 (1991).

${ }^{6}$ H. S. Sozuer, J. W. Haus, and R. Inguva, Phys. Rev. B 45, 13962 (1992); J. Opt. Soc. Am. B 10, 296 (1993).

${ }^{7}$ P. R. Villeneuve and M. Piche, Phys. Rev. B 46, 4969 (1992); 46, 4973 (1992).

${ }^{8}$ R. D. Meade, K. D. Brommer, A. M. Rappe, and J. D. Joannopoulos, Appl. Phys. Lett. 61, 495 (1992).

${ }^{9}$ M. Plihal, A. Shambrook, A. A. Maradudin, and P. Sheng, Opt. Commun. 80, 199 (1991); M. Plihal and A. A. Maradudin, Phys. Rev. B 44, 8565 (1991).

${ }^{10}$ E. Yablonovitch, T. J. Gmitter, R. D. Meade,
A. M. Rappe, K. D. Brommer, and J. D. Joannopoulos, Phys. Rev. Lett. 67, 3380 (1991).

${ }^{11}$ R. D. Meade, K. D. Brommer, A. M. Rappe, and J. D. Joannopoulos, Phys. Rev. B 44, 13772 (1991).

${ }^{12} \mathrm{~S}$. L. McCall, P. M. Platzman, R. Dalichaouch, D. Smith, and S. Schultz, Phys. Rev. Lett. 67, 2017 (1991); S. Schultz and D. R. Smith (unpublished).

${ }^{13}$ W. Robertson, G. Arjavalingan, R. D. Meade, K. D. Brommer, A. M. Rappe, and J. D. Joannopoulos, Phys. Rev. Lett. 68, 2023 (1992).

14 J. B. Pendry and A. MacKinnon, Phys. Rev. Lett. 69, 2772 (1992).

${ }^{15}$ M. Sigalas, C. T. Chan, K. M. Ho, and C. M. Soukoulis (unpublished).

${ }^{16}$ A. MacKinnon and B. Kramer, Phys. Rev. Lett. 47, 1546 (1981); Z. Phys. B 53, 1 (1983); A. MacKinnon, ibid. 59, 385 (1985).

${ }^{17}$ C. M. Soukoulis, I. Webman, G. S. Grest, and E. N. Economou, Phys. Rev. B 26, 1838 (1982); A. D. Zdetsis, C. M. Soukoulis, E. N. Economou, and G. S. Grest, ibid. 32, 7811 (1985); C. M. Soukoulis and G. S. Grest, ibid. 44, 4685 (1991).

${ }^{18}$ J. B. Pendry, A. MacKinnon, and P. J. Roberts, Proc. R. Soc. London Ser. A 437, 67 (1992). 